\title{
In-vitro sperm immobilization activity and biocompatibility study of NVD terpolymer
}

\author{
Sachin Gupta ${ }^{1 *}$, Ruchi Yadav², Mayank Thakur ${ }^{1}$
}

${ }^{1}$ Department of Pharmacology, Advance Institute of Biotech and Paramedical Sciences, Kanpur, India

${ }^{2}$ Department of Community Medicine, Uttar Pradesh University of Medical Sciences, Saifai, India

Received: 08 April 2017 Accepted: 17 April 2017

*Correspondence to:

Mr. Sachin Gupta,

Email:

sachincsjm@rediffmail.com

Copyright: (C) the author(s), publisher and licensee Medip Academy. This is an openaccess article distributed under the terms of the Creative Commons Attribution NonCommercial License, which permits unrestricted noncommercial use, distribution, and reproduction in any medium, provided the original work is properly cited.

\begin{abstract}
Background: Boronate derivatives have been used in affinity chromatography for separation of cells based on their glycoprotein content. Boronate containing polymers when used intravaginally before sexual intercourse could bind to the glycoproteins present on sperm cell surface and render them immobile, which potentially may work as female-controlled contraceptive. To study this hypothesis NVD terpolymer which contains boronic acid was studied on goat sperm and its biocompatibility was accessed on NIH3T3 fibroblast.

Methods: Sperm motility study was carried out on goat sperm cells. The study was divided into two groups, test group (NVD terpolymer in simulated vaginal fluid) and negative control (simulated vaginal fluid only) performed using Sander-crammer assay. In the test group, the study was started from $0.1 \%$ of the polymer solution, until the half of the sperms became non-motile as compared to normal control. The biocompatibility study was performed by culturing the NIH3T3 fibroblast with different concentrations of NVD polymer, followed by cell viability assay by performing 3-(4, 5-dimethylthiazol-2-Y1)-2, 5-diphenyltetrazolium bromide (MTT) assay at 6, 12 and 24 hour spectrophotometrically.

Results: Sander-crammer assay resulted in significant (P-value < 0.05) decrease in motile sperm count in test group when compared to control. At $7.5 \%$ concentration, the half of the sperms rendered immobile, and this was termed as effective concentration 50 (EC50). In-vitro biocompatibility study using NIH3T3 fibroblasts culture and MTT assay with cultured cells at 6, 12 and 24 hour, revealed that the polymer is biologically compatible as there were no significant change (P-value $<0.05)$ in the absorbance.

Conclusions: Boronate containing polymer, such as NVD terpolymer has invitro sperm immobilizing activity in goat sperm model, with further research in this area could yield a potential female control contraceptive agent.
\end{abstract}

Keywords: Boronate polymer, Contraceptive, Sander-crammer assay, Sperm immobilization

\section{INTRODUCTION}

What strikes in the mind of a woman before having sexual intercourse, as the consequence of intercourse she should not conceive? As an unplanned pregnancy might have the impact on aspects of her life, like education, finance, future goals, relationships, social life and her mental and physical health. It has seen that the promiscuous adolescents as well as adults, most often engage in 'ad hoc' relationships which involve sexual contacts, wherein women give more priority to pregnancy protection over sexually transmitted diseases (STDs) prevention while choosing contraceptive methods. In that, an unwanted pregnancy is the biggest fear which is even more important that getting any sexually transmitted diseases. $^{1}$

Hence, the protective methods were used provided only pregnancy protection. ${ }^{2}$ Condoms are the most effective in preventing the occurrence of pregnancies as well as STDs but are not controlled and accepted by females, along with the problem of their safe disposal. ${ }^{3}$ Gonorrhea, syphilis, and chancroid are considered typical sexually transmitted disease, but apart from these other pathogens 
are also transmitted through sexual intercourse such as Herpes simplex virus type 2 (HSV-2), Chlamydia trachomatis, human papillomaviruses (HPV), which require attention while developing a contraceptive. Adolescent women are more prone to STDs, of which many remain asymptomatic favoring the spread of disease. $^{4}$ It has been a big global concern that over 200 million pregnancies occur every year and of which $50 \%$ of are unwanted. There was large no. of contraceptives available in the market but successful prevention of pregnancy is major objective and the selection of a contraceptive method depends upon product's acceptability and couple's willingness. ${ }^{5}$

A recent study about the contraceptive need and use in developing countries showed that the number of women wanting to avoid pregnancy has increased along with the increase in the demand for modern contraceptive methods. ${ }^{6}$ Spermicides are among these contraceptives agents and are effective in preventing the occurrence of pregnancy, but their repeated use causes cervical irritation and damage to normal vaginal microflora which protects from various pathogens. Currently marketed vaginal contraceptives preparations contain surfactants as an active agent, among which most commonly used one is nanoxynol-9 (N-9). Frequently use of nonoxynol-9 is reported to cause vaginal epithelium damage, which in term increases risk of HIV acquisition. ${ }^{7}$ Hence, there is the need to search for a suitable and widely acceptable contraceptive choice that fulfills the needs of a larger group. Previously, Azadirachta indica leaf extract, and a peptide from an amphibian skin, Dermaseptin S4, and its derivatives, has been shown as an efficacious spermicide, as well as antimicrobial activity. ${ }^{8,9}$ Also, the ethanolic extract of Ensete superbum seeds has shown the spermicidal activity on human sperms in vitro. ${ }^{10}$ Various polymers, such as anionic polymeric hydrogels were noted to decrease the sperm motility, this motivated to relook in this area. ${ }^{11}$

Boronate derivative such as phenyl boronate has the unique property to form reversible covalent interaction with compounds like carbohydrates, nucleotides, nucleosides, catechols etc. and hence used as the ligand in affinity chromatography for separation of abovementioned compounds. In boronate affinity chromatography the interaction of boronate with various cis-diol containing compounds such as carbohydrates, nucleosides, nucleotides, glycoproteins, etc, takes place resulting in the formation of the cyclic ester. The major structural requirement for boronate interaction is that the two hydroxyl groups of diols should be adjacent to the carbon atom in the coplanar configuration. ${ }^{12}$ Boronate affinity matrices developed initially for separation of carbohydrates and nucleic acids. ${ }^{13}$ Later on, this technique was further explored for separation of the wide range of cis-diol compounds and neoglycoproteins, yeast cells were separated by using poly (HEMA-co-VPBA) grafted cryogel matrices. ${ }^{14,15}$ NVD terpolymer is a copolymer and stands for $\mathrm{N}$-isopropylacrylamide-co-4 vinylphenylboronicacid-co-2-(dimethylamino) ethyl methacrylate terpolymer. It was synthesized and patented by Indian Institute of Technology, Kanpur. Since NVD contains NIPAM; it also has the property to become gel around $32^{\circ} \mathrm{C}$. One of the advantages of becoming of the gel is that it will remain at the site of action for prolonged time than the solution form. ${ }^{16}$ Hence, the objective of this study was to evaluate the effect of NVD terpolymer on goat sperm motility, based upon its interaction with cisdiol containing moieties present on sperm cell, to find it as a novel contraceptive method.

\section{METHODS}

\section{Reagents and material}

\section{Simulated vaginal fluid}

Vaginal fluid simulant was prepared by dissolving sodium chloride $(3.51 \mathrm{~g})$, potassium chloride (1.40 g), calcium hydroxide $(0.222 \mathrm{~g})$, bovine serum albumin (Sigma Aldrich, United States) (0.018), lactic acid (2.0 $\mathrm{g}$ ), acetic acid glycerol $(1.0 \mathrm{~g})$, urea $(0.4 \mathrm{~g})$ and glucose $(5 \mathrm{~g})$ in $1000 \mathrm{ml}$ of deionized water and the $\mathrm{pH}$ of mixture was adjusted to 4.2 using hydrochloric acid $(\mathrm{HCl})$ and sterilized by autoclave. ${ }^{17}$

\section{Phosphate buffer saline}

Phosphate buffer saline (PBS) was prepared by dissolving $8 \mathrm{~g}$ of sodium chloride (Loba Chemie, India), $0.2 \mathrm{~g}$ of potassium chloride (Loba Chemie, India), $1.44 \mathrm{~g}$ of disodium hydrogen phosphate (SRL, India), $0.24 \mathrm{~g}$ of potassium dihydrogen phosphate (Thermo Fisher Scientific, United States) in $1000 \mathrm{ml}$ of deionized water and $\mathrm{pH}$ of the solution was adjusted to 7.4 and sterilized by autoclave.

\section{Dulbecco's modified Eagle's medium (DMEM)}

DMEM (Sigma Aldrich, United States) contains approximately four times as much of the vitamins and amino acids present in the original formula and two to four times glucose than the original. Additionally, it contains iron and phenol red. DMEM is suitable for most types of cells, including human, monkey, hamster, rat, mouse, chicken and fish.

\section{Polymer}

NVD terpolymer was synthesized in Department of Biological Science and Bioengineering (BSBE), Indian Institute of Technology (IIT), Kanpur and provided as gift sample to screen the sperm immobilization activity. ${ }^{18}$

\section{Goat sperm}

Goat sperm was obtained from newly sacrificed male goat from their slaughterhouse. As the goat is a mammal, its sperm can be used to observe the effect of polymers. 


\section{Cell line}

NIH3T3 Fibroblasts (NCCS, Pune), which is a standard cell line derived from mouse.

\section{Isolation of caudal goat sperm exudates}

Sperm was collected from the adult male goat. The goat testes were obtained from newly sacrificed male goat through Kanpur, from their slaughterhouse. The sac was cut and cauda epididymides were dissected out of the testis, and the caudal region was identified and picked carefully, avoiding any blood vessel rupture. The caudal regional was put into a petri dish containing $5 \mathrm{ml}$ of Phosphate buffer saline (PBS) medium and cut into several pieces. Sperms come out into the PBS medium and the count was performed using a hemocytometer. The temperature of the PBS medium containing sperms was maintained at $37^{\circ} \mathrm{C}$ using the water bath. Sperm suspensions having highly motile sperm, whose count 20 $\mathrm{X} 105 / \mathrm{ml}$ were used for the studies.

\section{Preparation of NVD terpolymer dilutions}

Different concentrations of NVD terpolymer were prepared by dissolving the polymer using simulated vaginal fluid so that the experiment can be simulated to physiological parameters. As the polymer application site will be the vagina, so that it is rational to screen in the environment as that of the vagina.

\section{Sperm immobilization assay (Sander-Cramer assay)}

Sperm immobilization assay was performed following Sander-Crammer assay. ${ }^{19}$ The study was conducted in two groups, first the normal control group and another test polymer group. The test was performed with different concentrations of NVD terpolymer starting from $0.1 \%$ until the decrease in motile sperm count came to approximately 50\% (EC50) was arrived by following the modified method of Sander-Crammer assay. Different concentrations of the NVD terpolymer were prepared by dissolving the NVD terpolymer in simulated vaginal fluid (SVF), so as to simulate the test conditions as that of vagina. In the test group, $0.1 \mathrm{ml}$ of goat sperm suspension was added to $0.1 \mathrm{ml}$ of different concentrations of NVD terpolymer solution and vortexed (at very low speed) for 5-10 seconds. An aliquot of $0.02 \mathrm{ml}$ was immediately placed on Neubauer's hemocytometer, covered with a cover glass and examined under a microscope (Nikon YS100, Japan). Motility of sperm cells was counted as per World Health Organization (W.H.O.) manual for semen analysis. In control group, $0.1 \mathrm{ml}$ of simulated vaginal fluid (SVF) was added to $0.1 \mathrm{ml}$ of goat sperm suspension. Each analysis was conducted in triplicates. Means of triplicate determinations and their standard deviation were plotted.

Percentage decrease in motility was determined using following formula:
$\%$ Decrease in motility $=[$ (No. of motile sperm in control-No. of motile sperm in test)/(No. of motile sperm in control) $] \times 100$.

\section{In-vitro cytotoxicity assay}

MTT assay is used often in the measurement of cell viability and proliferation forms the basis for several in vitro assays of a cell population reaction to external elements. The yellow colored tetrazolium MTT (3-(4, 5dimethylthiazolyl-2)-2, 5-diphenyltetrazolium bromide) dye is reduced by metabolically active cells, in part by the action of enzymes called dehydrogenase, to produce reducing equivalents such as NADH and NADPH. The resultant intracellular purple formazan can be solubilized and measured by spectrophotometric methods.

The colorimetric MTS (3-(4, 5-dimethylthiazol-2-yl)-5(3-carboxymethoxy-phenyl)-2-(4-sulfophenyl)-2-

htetrazolium) in-vitro assay is an upgraded version of the validated MTT method. MTS assay has the advantage of being soluble and hence no solubilisation step is required in this method. ${ }^{20}$

\section{NIH $3 T 3$ culture}

Cell culture testing of NIH-3T3 fibroblast cells was conducted to perform biocompatibility testing of NVD terpolymer. NIH3T3 fibroblasts whose concentrations were $1 \times 105 / \mathrm{ml}$ were taken into 48 well-treated plates and seeded in DMEM, 10\% FBS and antibiotics. Different concentrations of NVD polymer such as $0.05 \%, 2.5 \%$, $5 \%$, and $7.5 \%$ were prepared in PBS and put into respective wells. Control was taken in which only PBS was added. It was allowed to grown at $37^{\circ} \mathrm{C}, 5 \% \mathrm{CO}_{2}$, and 95\% R.H. MTT cytotoxicity assays were performed to evaluate cellular behaviour on the NVD terpolymer. ${ }^{21}$

\section{MTT assay}

MTT assay was performed using tetrazolium dye and absorbance of each set of the solution was taken at 570 $\mathrm{nm}$ using a spectrophotometer. Absorbance was taken at 8 hour, 16 hour and 24 hour after incubation of the NIH3T3 fibroblasts. All the experimental groups were performed in triplicates in order to observe the reproducibility of the test results.

\section{RESULTS}

\section{In vitro sperm immobilization assay (Sander-Cramer assay)}

Various concentrations of NVD terpolymer starting from $0.1 \%$ until the motile sperm count was reduced to half to that of control were screened on goat sperm. The concentration at which the motility remained half was termed as effective concentration 50 (EC50). Test polymer concentration, at which the effective concentration 50 (EC50) arrived was $7.5 \%(7.5 \mathrm{mg} / \mathrm{ml}$ 
NVD terpolymer) and also it was reported significant (Pvalue $<0.05)$ decrease in the total motile sperm count. Hence, the effective concentration was $7.5 \%$. In both, normal controls, as well as test polymer group, the mobile as well as nonmotile sperm cells, were counted using Neubauer's hemocytometer under the microscope. For each group, all four chambers, each chamber containing 16 sub-blocks were counted for the sperm count. The numbers of mobile, nonmotile and total sperms were calculated from the data gathered. The data was generated from Microsoft excel. By performing the sperm immobilization assay it was seen that the NVD terpolymer reduces the motility as the no. of motile sperm in test groups are significantly lower than the no. of sperm in the normal control group. In the normal control group, the mean sperm count is $56.72 \mathrm{X} 105 / \mathrm{mL}$, whereas in NVD polymer treated group it is $28.33 \mathrm{X} 105 / \mathrm{ml}$. This is plotted in Figure 1. The percentage decrease in the motility is calculated by this formula.

$\%$ Decrease in motility $=[(56.72-28.33) /(56.72)] \times 100$ $=50.05 \%$

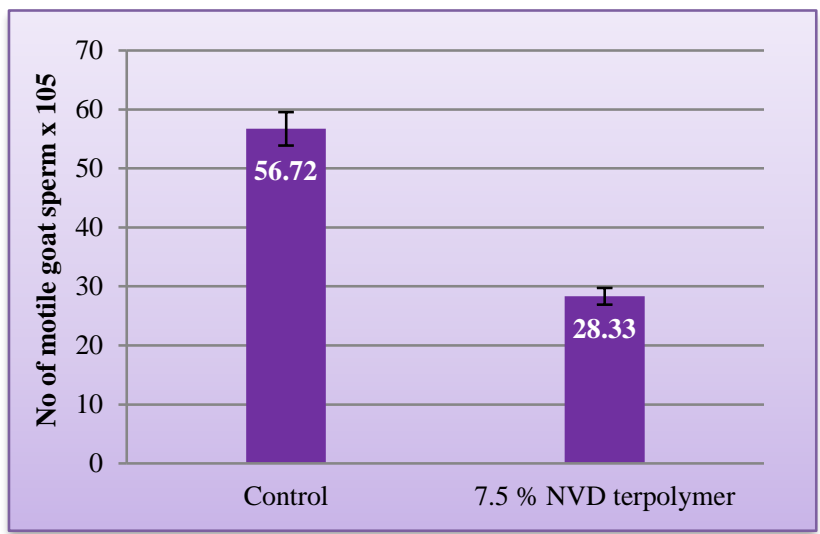

Figure 1: Comparison between normal control and test polymer (NVD-7.5 mg/ml).

The effective concentration 50 (EC50) at which the half of the sperms were rendered immobile was found to be $7.5 \%$ as compared to that of control. This concentration was repeated three times to check the reproducibility of the test.

\section{In-vitro cytotoxicity assay (MTT Assay)}

The MTT assay was performed for the assessment of the polymer's toxicity profile on NIH3T3 fibroblasts cells. Its procedure was performed as described in materials and method section. Each group of the test substance, as well as normal control, was taken in triplicates and absorbance was measured at 8,16 and 24 hour at $570 \mathrm{~nm}$. The data was gathered and mean as well as standard deviation were calculated. The results are dissipated in the form of bar diagram in Figure 2, 3, and 4 for 8 hour, 16 hour, and 24 hour, respectively. The cell viability experiment reveals that the fibroblast cells are able to freely grow even in presence of different concentrations of the NVD polymer.

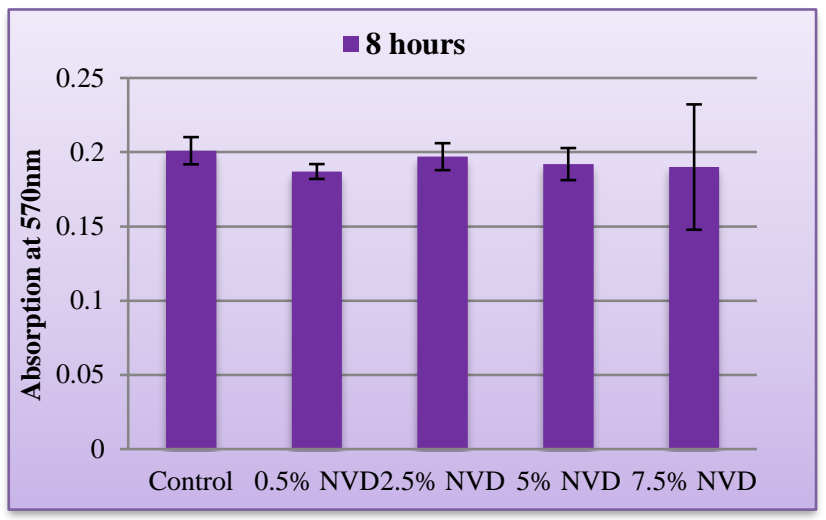

Figure 2: Cell viability (MTT) assay at 8 hour.

There was no significant ( $\mathrm{P}$-value for this experiment was $<0.05)$ change in the test groups and normal control. The test to know significant/insignificant was performed by comparing the normal control and different polymer concentration.

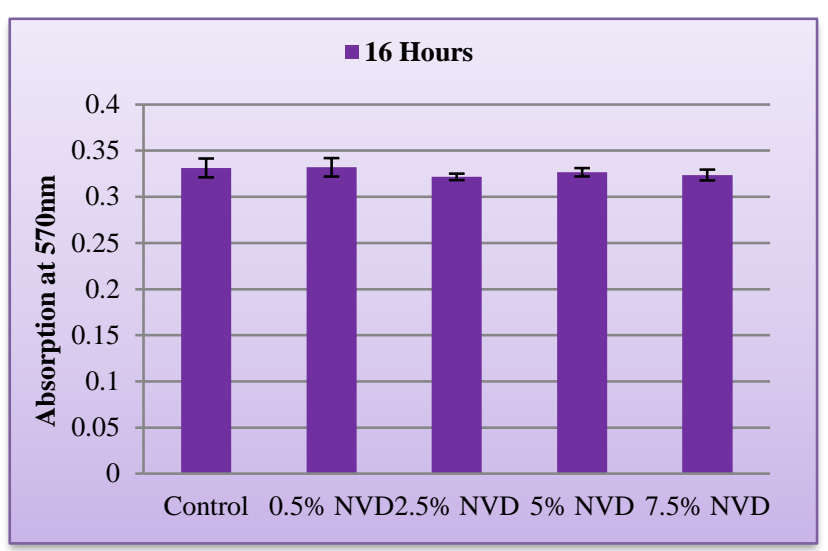

Figure 3: Cell viability (MTT) assay at 16 hour.

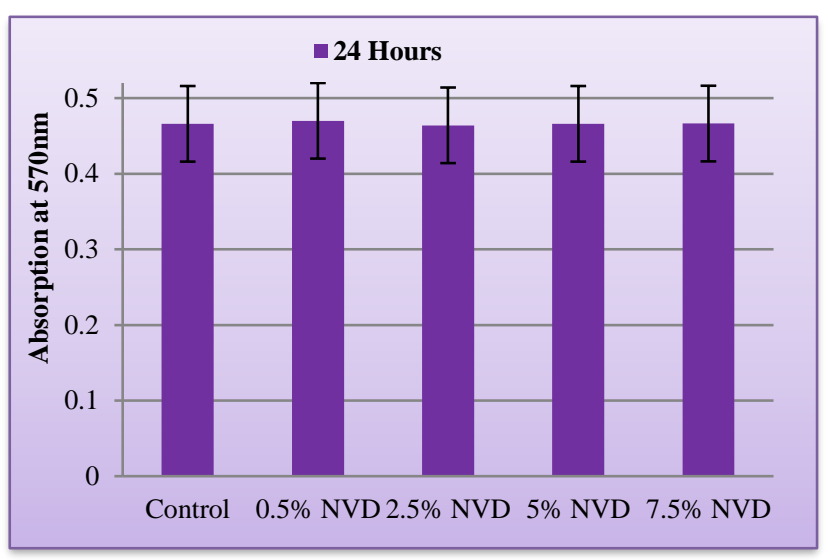

Figure 4: Cell viability (MTT) assay at 24 hour.

\section{DISCUSSION}

Unwanted pregnancy is the bigger fear among promiscuous adolescents over the sexual transmitted diseases while choosing a contraceptive method. ${ }^{1}$ Condoms are the most effective in preventing the occurrence of pregnancies as well as STDs but are not 
controlled and accepted by females. ${ }^{3}$ Hence, there is a need to search for widely acceptable short term contraceptive methods. As stated earlier that boronate containing polymers have been successfully used for separation of cells based upon their glycoprotein content. ${ }^{12}$ Similarly NVD terpolymer, which is a boronate containing polymer, may bind to glycoproteins present on the sperm cell surface and this interaction could render them immobile and may find application as female controlled contraceptive.

In order to find effective as well as safe sperm immobilizing agent, different experimental models have been performed. Sander-Crammer assay has been considered useful by many for evaluation of spermicidal effects of drugs. ${ }^{19}$ The current study was conducted by dissolving the NVD terpolymer in simulated vaginal fluid so as to simulate the intravaginal test conditions. ${ }^{17}$

Different concentrations of NVD terpolymer were prepared and a fix amount $0.1 \mathrm{ml}$ of the polymer solution was added to an Eppendorf containing $0.1 \mathrm{ml}$ of goat sperm suspension, which was the vortexed for 5-10 seconds. An aliquot of $0.02 \mathrm{ml}$ was then placed on Neubauer's hemocytometer and examined under microscope for motile and non-motile goat sperms. In the test group, the study was started from $0.1 \%$ of the polymer solution until the half of the sperms became nonmotile as compared to normal control. At $7.5 \%$ concentration, the half of the sperms rendered immobile. As previously mentioned that boronate containing polymer form cyclic ester bond with cis-diol containing compounds such as nucleotides, nucleosides, glycoproteins, might also interact in similar fashion with the glycoproteins present on sperm cell surface, which was the main objective of the study to understand the probable mechanism. ${ }^{12}$ Looking at the results, it indicates that the resultant effect on sperm cell motility would have been resulted from the expected cis-diol interaction between the abundantly present glycoproteins on sperm cell and the boronic acid moiety present in NVD terpolymer, as postulated. In biocompatibility study, different concentrations of the test polymer, $0.05 \%, 2.5$ $\%, 5.0 \%$, and $7.5 \%$ were cultured with NIH3T3 fibroblast in DMEM medium and a blank control (without polymer) was taken for reference and the samples were taken at 8, 16 and 24 hour interval and MTT assay (cell viability assay) was performed, and the absorbance of test samples were recorded using spectrophotometer at 570 $\mathrm{nm}$. The absorbance of the different concentrations of the test groups was compared with control using Microsoft Excel, and there were no significant $(\mathrm{P}$-value $<0.05)$ changes noted.

\section{ACKNOWLEDGEMENTS}

Authors acknowledge Prof. Ashok Kumar, Department of Biological Sciences and Bioengineering, Indian Institute of Technology Kanpur, for providing an opportunity to work in his group, and all necessary reagents, test polymer (NVD terpolymer), equipment and facilities. Also, authors would acknowledge, Mr. Arun Kumar Teotia, for demonstrating various techniques. Further, authors extend their thanks to Dr. Ankur Gupta, and Mr. Samarjeet Singh, for always standing together.

\section{Funding: No funding sources}

Conflict of interest: None declared

Ethical approval: The study was approved by the Institutional Ethics Committee

\section{REFERENCES}

1. Minnis AM, Padian NS. Choice of female- controlled barrier methods among young women and their male sexual partners. Fam Plann Perspec. 2001;33:332834.

2. Jain RK, Maikhuri JP, Kumar STVS, Sharma VL, Dwivedi AK, Mitra K et al. Novel disulphide esters of carbotioicacid as potent, non-detergent spermicides with low toxicity to Lactobacillus and HeLa cells in vitro. Human Reprod. 2007;22:708-16.

3. McMohan S, Hansen L, Mann J, Sevigny C, Wong T, Roache M. Contraception. BMC Women's Health. 2004;4:S25.

4. Catalone BJ, Kish-Catalone TM, Neely EB, Budgeon LR, Ferguson ML, Stiller C et al. Comparative safety evaluation of the candidate vaginal microbicide C31G. Antimicro Agent Chemother. 2005;49:150920.

5. Hagga J, Tsui A, Wasser HJ. Reproductive health in developing countries: Expanding dimensions, building solutions. National Academy Press, Washington; 1997.

6. Darroch JE, Singh S. Trends in contraceptive need and use in developing countries in 2003, 2008, and 2012: an analysis of national surveys. Lancet. 2013;381:1756-62.

7. Piot D. Nonoxynol-9 not effective microbicide, trial shows. UNAIDS press release, 2000.

8. Khillare B, Shrivastav TG. Spermicidal Activity of Azadirachta indica (neem) leaf extract. Contracep. 2003;68:225-9.

9. Khaled H, Zairi A. In vitro spermicidal activity of peptides from amphibian skin: Dermaseptin S4 and derivatives. Bioorg Med Chem. 2008;16:266-75.

10. Sarwar G, Arif M, Khillare B, Thakur SC. In vitro study of the contraceptive spermicidal activity of Ensete Superbum on human sperm. Int J Pharm Pharmaceu Sci. 2014;8:205-7.

11. Singh H, Jabba MS, Ray AR, Vasudevan P. Effect of anionic polymeric hydrogels on spermatozoa motility. Biomaterials. 1984;5:307-9.

12. Srivastava A, Shakya AK, Kumar A. Boronate affinity chromatography of cells and biomolecules using cryogel matrices. Enzyme Microb Tech. 2012;51:373-81.

13. Angyal S, Greeves D, Pickles V. The stereochemistry of complex formation of polyols with borate and 
periodate anions, and with metal cations. Carbohydr Res. 1974;35:165-73.

14. Bergold A, Scouten WH. Boronate Chromatography. Solid Phase Biochem. 1983;34:149-87.

15. Li YC, Larsson EL, Jungvid H, Galaev IY, Mattiasson B. Separation of neoglycoproteins with different degree of glycosylation by boronate chromatography. Chromatographia. 2001;54:213-7.

16. Kumar A. Hydrogel scaffolds for tissue engineering. USPTO 2011, Publication number US8883503 B2.

17. Owen DH, Katz DF. Vaginal fluid stimulant. Contracep. 1999;93:91-5.

18. Reddy RM, Srivastava A, Kumar A. Monosaccharide-responsive phenylboronate-polyol cell scaffolds for cell sheet and tissue engineering applications. PLoS One. 2013;8(10):e77861.
19. Sander FV, Cramer SD. A practical method for testing the spermicidal action of chemical contraceptives. Human Fertil. 1941;6:134-53.

20. Mosmann T. Rapid colorimetric assay for cellular growth and survival: Application to proliferation and cytotoxicity assays. J Immunologic Methods. 1983;65:55-63.

21. Rodolfo V, Ortiz CR, Tapia S, Gonzalez G, Trillio E, Garza KM, Stafford SW et al. In vitro biocompatibility studies of fibroblast cells on Ti-Ta alloys. Materials Transactions. 2002;43:2991-94.

Cite this article as: Gupta S, Yadav R, Thakur M. In-vitro sperm immobilization activity and biocompatibility study of NVD terpolymer. Int J Basic Clin Pharmacol 2017;6:1221-6. 